\title{
Study on Half Shaft Bushing Punching Slug Defect and Process Optimization
}

\author{
Li-ying Liu ${ }^{1 *}$, Wan-gui $\mathrm{Yao}^{2}$, and Pan $\mathrm{Li}^{3}$ \\ ${ }^{1,2,3}$ Liying Liu, Beijing Research Institute of Mechanical \& Electrical Technology, China \\ ${ }^{*}$ Corresponding author
}

Keywords: Punching Process; Half Shaft Bushing; Numerical Simulation; Process Optimization

\begin{abstract}
Half shaft bushing is the important parts of automobile axle housing. Slug is a very typical defect in metal punching progress. In this dissertation, the punching slug forming process has been simulated by DEFORM-3D software, and related causes have been analyzed. To optimize the process data for punching process and ensure the appropriate thickness of the slug, BP neural network combined with genetic algorithm is applied. Qualified half shaft bushing has been produced with the process data after optimizing in the practical experiment. The research results are of great worth for diminishing the half shaft bushing forming defects and making the reasonable technological procedure.
\end{abstract}

\section{Introduction}

Driven axle is the second core assembly after engine and cab in commercial vehicle[1]. As the most important safety component in commercial vehicle, half shaft bushing bears not only its body and cargo weight, but also the alternating stress and torque under different road condition. Therefore, high quality half shaft bushing is a significant guarantee for driving security of commercial vehicle[2].

In this paper, DEFORM-3D is used to simulate the forming process of half shaft bushing. The causes of forming defect in the pipe end are analyzed and the reasonable solutions are put forward combined with engineer test. The research result will provide some useful instruction to deal with punching slug and guide the practical production.

\section{Hot Extrusion Forming Process of Half Shaft Bushing}

As is shown in Fig.1, this product has a hollow variable diameter and section tube shape, high slenderness ratio. There is a flange adapter in the long diameter end. So it is hard to produce and the forming precision is difficult to guarantee.

The main process is upsetting, punching, punching and forward extrusion, upset-extruding flange till finishing all the forming tasks, the operation as shown in Fig.2. Firstly, heat the round bar stock to forging temperature by hot rolling, then form the thorough inner hole and outside contour accurately and there is no need of setting up the stamping or cutting head process.

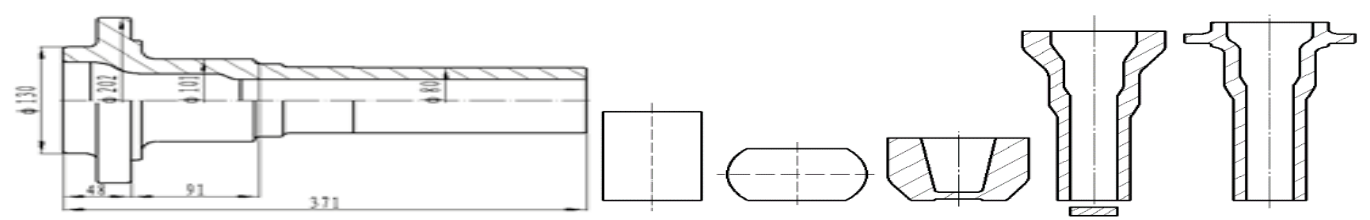

Fig.1 Half shaft bushing

Fig.2 Round bar, upsetting, punching and upset-extruding flange, forward extrusion

\section{Punching Slug Defect}

In the piercing and forward extrusion process, the appropriate length core bar is used to remove the slug after closed punching, then the inner hole is extruded forwardly. Design a set of terrace die to realize this goal, which is composed of ring punch and core bar. After that ring punch is extruded 
forwardly, the core bar could remove the slug. If the slug is not removed in the debugging process, which means that the connection between the slug and pipe wall is not break, slug will occur in the outside wall of punch when core terrace die goes down, as shown in Fig. 3. If the slug is not removed, the metal flow will be blocked in the end of half shaft bushing and the upset-extruding can not go on. If the slug is removed forcefully, it will cause serious gap and metal waste in the end of pipe.

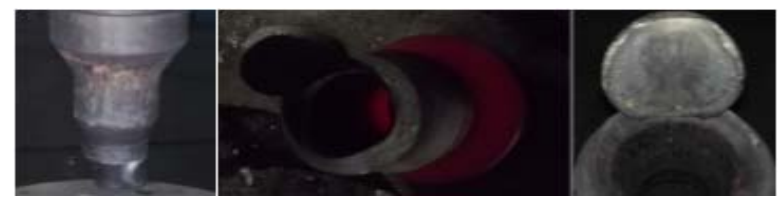

Fig.3Punchingslug adheres to the tube end

\section{The Cause Analysis of Slug}

Slug adheres to pipe wall is due to non-uniformity of metal flow while the attachment of slug and core bar is being pushing out. The slug is thrust by shearing action while core bar down. If the rim of slug deforms non-uniformly, thrust at different time, a certain part of slug will break at first, and the crack will occur along the core bar until disrupting and dropping out. When the slug is too thick, the expanding of crack will be very slow. The unbroken part will drag slug for so long time that the action of the end of core bar could not guide the flow of whole metal. Some will be stuck and can not be divorced from restraint of pipe end or form the attachment part of slug. Although most of the slug is thrust by core bar, it will not drop down and adhere to the pipe end for restraint of retention metal.

There are many reasons caused parts of slug is first to break, mainly because the non-uniformity of metal organization near slug and gas effect. Gas pressure goes up dramatically in cavity of billet while core bar down. For that, the relative weak joints between slug and pipe wall will be the first to crack and release gas. The slug is restricted by the metal outside when it breaks out core bar. The thicker of slug, it is easier to form big flash to stretch slug. The more serious restraint effect of slug, it is harder to be flushed. In addition, while punching and forward extrusion process, punch eccentricity may also cause slug defect.

\section{The Finite Element Modeling of Slug Defect}

Thick slug is simulated by volume forming finite element software DEFORM-3D, based on the thermal coupling rigid visco-plastic finite element technology, and verified the causes of slug further.

Construct the 3Dmodel of round bar, upsetting, punching and upset-extruding flange, forward extrusion by Solid Works and choose the appropriate experiment condition.

When the press is punching the bar after upsetting, insufficient downstream distance of upper slider will lead to thick slug of punched billet on account of misoperation. In order to describe the influence of too thick slug, we simulate the distance of control terrace die. Other conditions remain unchanged, the thickness of slug is $35 \mathrm{~mm}, 25 \mathrm{~mm}$ and $15 \mathrm{~mm}$ respectively to simulate the punching and forward extrusion processes. The results of simulations has shown that the 35 and $25 \mathrm{~mm}$ slugs has not been washed away, however, the $15 \mathrm{~mm}$ one is flushed easily, as shown in Fig.4.

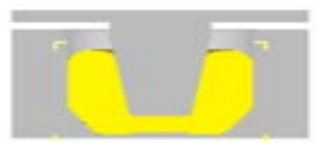

Thick of slug=35 $\mathrm{mm}$


Thick of slug= $25 \mathrm{~mm}$
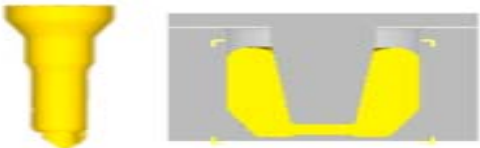

Thick of slug $=15 \mathrm{~mm}$

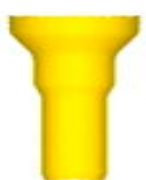

Fig.4Punching situations with different punching wad thicknesses 


\section{Optimization of Punching Process Parameters}

From the above analysis, we may safely draw the conclusion that over thickness of slug is the main reason that it can not be washed away. For half shaft bushing, we can judge that thickness of slug should be controlled in $25 \mathrm{~mm}$ from the simulation results. How to select a relatively appropriate slug thickness is vital to enhance the stock utilization, reduce energy consumption and save the cost of production. To avoid the trial and error to search optimization, this paper adopts BP neural network combined with genetic algorithm to confirm the best thickness slug.

The operation condition of die is the key factor affected the service life, among them force loading is the most significant one, under the defined structure, material and manufacturing process. Slug is formed in punching and force loading of punch must be taken full consideration in order to confirm the optimal slug thickness. It decides the distance of punch, force loading of punch is influenced by preheating temperature of die, initial forging temperature of billet and falling velocity of punch. This article designs orthogonal simulation test on billet punching process about different process parameter, to study force loading of punch as shown in Table.

Table 1 Factor and level

\begin{tabular}{|c|c|c|c|c|}
\hline Level & $\begin{array}{c}\text { Initial Forging } \\
\text { Temperature }\left[{ }^{\circ} \mathrm{C}\right]\end{array}$ & $\begin{array}{c}\text { Preheating } \\
\left.\text { Temperature[ }{ }^{\circ} \mathrm{C}\right]\end{array}$ & Falling Velocity $\left[\mathrm{mm} \cdot \mathrm{s}^{-1}\right]$ & Slug Thickness $[\mathrm{mm}]$ \\
\hline 1 & 1050 & 200 & 20 & 6 \\
\hline 2 & 1100 & 250 & 30 & 12 \\
\hline 3 & 1150 & 300 & 40 & 18 \\
\hline
\end{tabular}

Optimize the above process data by BP neural network combined with genetic algorithm, then gain the best slug thickness under specific condition.

First of all, determine the neural network structure according to experiment condition and result, secondly, use the punching process parameter as input data, maximum force of punch as output data to train the structure, then it can predict the experiment result under definite condition. Finally, we can deduce the best experiment result and its optimal experiment condition by using experiment condition as population individual in genetic algorithm and experiment result neural network forecasted as individual fitness values[3].

Write BP neural network combined with genetic algorithm program file based on stimulated data and optimize by MATLAB[5,6]. Choose 4-10-1 as BP neural network structure, 30times as iterations, 4 as population size, 0.4 as crossover probability, 0.2 as mutation probability, 4 as the length of the individual, use float-point encoding, to get the optimal results $272.5 \mathrm{t}$, the corresponding experiment condition is [1090.5 224.7 30.6 13.3]. This result can offer reference to select the best experiment condition and integrate the experiment condition data, determine the final punching experiment parameter. The simulation results show that the max force is about $265 \mathrm{t}$.

To offset the heat loss in the air and ensure the temperature parameter, heating temperature of billet and reheating temperature of die should be higher than the regulated parameter according to the new process parameters for debugging.

\section{Experimental Confirmation}

Align the terrace die and cavity die in every process and limit the eccentricity error in $0.2 \mathrm{~mm}$. Initial forging temperature before upsetting is $1150{ }^{\circ} \mathrm{C}$, preheating temperature of punching die is $250{ }^{\circ} \mathrm{C}$, use Graphite emulsion as lubricant, surface temperature of billet after upsetting should be $1100^{\circ} \mathrm{C}$, the slug thickness should be $13 \mathrm{~mm}$ by controlling the distance of terrace die, the max load of upper die is $260 \mathrm{t}$ shown in instrument, which is very close to predicted result. A qualified half shaft bushing is shown in Fig. 11 after slug is flushed in punching and forward extrusion process and flange is upset-extruding. The surface of sample is in the same level, slug tearing slits distributes equally, the flash is small, which declares that the design of slug thickness is reasonable. 


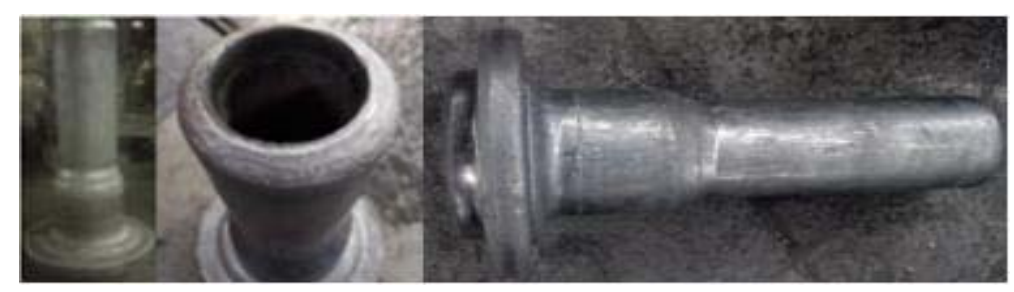

Fig.11 Produced qualified product of half shaft bushing

\section{Conclusions}

(1) This paper aims at finding the reason caused slug while debugging half shaft bushing forming process. And the main reason is that the thickness of slug is not appropriate. The numerical simulation is carried out for the problem, and the simulation results reflect the actual situation very well.(2) BP neural network combined with genetic algorithm is utilized to optimize the punching process parameter from the point of view of the mold force, and the appropriate slug thickness for production is obtained. This method reduces the artificial test error effectively and improves the efficiency of the commissioning, which is of great importance to remove slug and ensure the final forming of shaft. Qualified half shaft bushing has been produced with the process data after optimizing in the practical experiment and the reliability of the solution is proved.

\section{References}

[1]Zhang C B. Lightweight Optimization Study on 1141 Heavy Truck Driving AxleHousing[D]. Wuhan, Wuhan University of Technology, 2010.

[2] Steven W Bradley, Walter L Bradley. Analysis of failure of axle housing of crane truck with fracture mechanics[J], Engineering Failure Analysis, 1995, 2(4): 233-246.

[3]Shi F, Wang X C, Yu L, et al. 30 Case Analysis of MATLAB Neural Network[M]. Beijing: Beihang University Press, 2010.

[4] Howard Demuth, Mark Beale. Neural network toolbox for use with MATLAB[J]. Matlab Users Guide the Math Works, 2000,21(15);1225-1233.

[5] Robert J, Van Eyden. The Application of Neural Networksin the Forecasting of Share Prices[M]. Berlin: Finance and Technology Publishing, 1996. 